Results The overall level of obesity among Egyptian women rises from $30 \%$ in 1995 (urban=33\%; rural $=27 \%$ ) to $40 \%$ in 2008 (urban $=43 \%$; rural=34\%). Among urban women, in 1995, the prevalence of obesity is lower in the group without education $(24 \%$; $95 \%$ CI 19 to 29) in comparison to the group with secondary education (33\%; 95\% CI 29 to 37). In 2008, the prevalence of obesity has risen in a statistically significant manner in both groups compared with 1995. In addition, the prevalence in the group without education (45\%; 95\% CI 41 to 50 ) appears to have exceeded the prevalence in those with secondary education (41\%; $95 \%$ CI 38 to 44). Although there is overlap in the CI at the $95 \%$ level, the overall trend suggests that the social gradient in obesity may be reversing, as predicted elsewhere.

Conclusion Egypt provides a dynamic model of the reversal of the social gradient of obesity. Further analysis of Demographic and Health Surveys using other indicators of socio-economic status and risk factors for obesity such as consumption of fruit and vegetables may shed light on the processes behind the probable gradient reversal, and the factors putting the poor at increased risk of obesity. This is important in informing urgent prevention efforts at a population level.

\section{Policy \\ 050 NEWS MEDIA COVERAGE OF NICE'S DECISIONS ON NEW HEALTH TECHNOLOGIES}

doi:10.1136/jech.2010.120956.50

${ }^{1} \mathrm{H}$ Chauhan, ${ }^{1} \mathrm{~B}$ Dhesi, ${ }^{1} \mathrm{~N}$ Patel, ${ }^{1} \mathrm{~A}$ Uppal, ${ }^{1} \mathrm{~J}$ Mohammed, ${ }^{1} \mathrm{~A}$ Ahmad, ${ }^{2} \mathrm{~W}$ Greenheld, ${ }^{2} Y-F$ Chen. ${ }^{1}$ College of Medical and Dental Sciences, University of Birmingham, Birmingham, UK; ${ }^{2}$ Public Health, Epidemiology and Biostatistics, University of Birmingham, Birmingham, UK

Objective This project aims to: (1) describe the frequency of news coverage in mass media related to the National Institute for Health and Clinical Excellence (NICE) draft or final guidance; (2) analyse the types of evidence and sources of information that was quoted in the news; (3) compare whether the patterns of coverage differ between media.

Design A survey of news articles related to decisions made by NICE's Health Technology Appraisal committees was conducted. Relevant news articles were retrieved from websites of major UK news media. Inclusion criteria were: (1) news articles related to specific NICE decision(s); (2) articles were written by a reporter/ writer/editor of the news media. Articles that mentioned NICE for other reasons, columns and readers' letters were excluded.

Setting Major UK news media, including national newspapers and news channels.

Samples News articles published during 2007-2008 in 13 different sources of media including tabloids (Daily Express, Daily Mail, News of the World, The Mirror, The Sun), broadsheets (Independent, Guardian, The Telegraph, The Times) and news channels (BBC, ITN, Sky News and Reuters).

Main outcome measure The following data were collected by one author and checked by another: nature of guidance (disease area; positive or negative recommendation), use of generic or brand name and source/type of evidence that was quoted. Descriptive statistics were compiled and comparisons between types/sources of news media were made using $\chi^{2}$ test.

Results 329 articles were included. BBC, Daily Mail and The Telegraph published more than 50 articles related to health technology appraisal whereas ITN and News of the World published less than 10 articles during the 2-year period assessed. Two-thirds (220/329) of the articles were related to negative recommendations. There was significant difference in the proportion of articles relating to negative recommendations between individual sources of media $(p=0.001)$ but not between types of media $(p=0.286)$. Cancer $(33 \%)$, neurology-mainly Alzheimer's disease (22\%), ophthalmology $(13 \%)$ and rheumatology $(10 \%)$ were most frequently covered areas. $58 \%(192 / 329)$ of the articles quoted only brand names without mentioning generic names of the drugs. Approximately $50 \%$ of articles included statements of effectiveness without referring to the source of evidence and another $40 \%$ did not describe clinical effectiveness. $24 \%$ of articles did not mention drug costs or cost-effectiveness.

Conclusion NICE decisions on new drugs, particularly negative recommendations, attracted significant media attention but the coverage and contents varied substantially between individual sources.

\section{A POLICY EFFECTIVENESS-FEASIBILITY LOOP? PROMOTING THE USE OF EVIDENCE TO SUPPORT THE DEVELOPMENT OF HEALTHY PUBLIC POLICY}

doi:10.1136/jech.2010.120956.51

${ }^{1} \mathrm{~N}$ Unwin, ${ }^{2} \mathrm{~K}$ Bennett, ${ }^{3} \mathrm{~S}$ Capewell, ${ }^{1} \mathrm{~J}$ Critchley, ${ }^{4} \mathrm{~F}$ Fouad, ${ }^{5} \mathrm{~A}$ Husseini, ${ }^{3} \mathrm{M}$ O'Flaherty, 4,6 W Maziak, ${ }^{7} \mathrm{~A}$ Mataria, ${ }^{1} \mathrm{P}$ Phillimore, ${ }^{8} \mathrm{H}$ B Romdhane, ${ }^{9} \mathrm{~B}$ Unal, ${ }^{1} \mathrm{~S}$ Zaman. ${ }^{1}$ Institute of Health and Society, Newcastle University, Newcastle-upon-Tyne, UK; ${ }^{2}$ Trinity Centre for Health Sciences, St James's Hospital, Dublin, Republic of Ireland; ${ }^{3}$ Department of Public Health, University of Liverpool, Liverpool, UK; ${ }^{4}$ Syrian Center for Tobacco Studies, Aleppo, Syria; ${ }^{5}$ Institute of Community and Public Health, Birzeit University, Palestine; ${ }^{6}$ School of Public Health, University of Memphis, Memphis, Tennessee, USA; ${ }^{7}$ Health Economics and Health Care Financing Unit, Division of Health Systems and Service Delivery, World Health Organization-Eastern-Mediterranean Regional Office, Cairo, Egypt; ${ }^{8}$ CVD Epidemiology and Prevention Research Laboratory, National Public Health Institute, Tunis, Tunisia; ${ }^{9}$ Department of Public Health, School of Medicine, Dokuz Eylul University, Turkey

Background Historically, policy initiatives have made variable contributions to improvements in public health. Today there is a growing interest in translating evidence from health research into healthy public policy. Although research evidence may be a component of policy development, it is rarely enough, because policy makers are subject to a wide range of influences. Furthermore, researchers and policy makers usually work within different time frames and rules of evidence. There is a growing, albeit limited, literature on how researchers can most effectively engage with policy makers. Evidence suggests that more active and effective dialogue between researchers and policy makers is needed, in the formulation of research questions, presentation of evidence, and drafting and choice of policy options.

Aim To develop, implement, and evaluate an interactive approach to informing policy for the prevention and management of cardiovascular disease (CVD) and diabetes.

Setting This work is being undertaken in four eastern Mediterranean territories, known to have high burdens of CVD and diabetes: Palestine, Tunisia, Turkey and Syria.

Methods and results Available epidemiological data are being identified, appraised and used to populate the IMPACT CHD Policy Model in order to examine CHD trends. Two corresponding epidemiological models have been developed to examine trends in type 2 diabetes and ischaemic stroke. An intensive review of the literature and consultation assists in the identification of efficacious policy interventions. A situation analysis is being undertaken within each country using mixed methods, which include: key informant and in depth interviews, document reviews, and participant observation. Its aim is to review current policy (stated and implemented), perceived facilitators and barriers to policy change, including health beliefs, and aspects of the health system. Policy makers are explicitly involved as key informants, participants, advisors and "lobbyists". The epidemiological modelling, evidence based reviews, and situation analyses are together being used to generate diverse policy 
options. The cost-effectiveness of each chosen option will then be assessed.

Conclusions We have developed a provisional framework for developing policy options, initially for the prevention of CVD and diabetes. This is based on local epidemiological data, an assessment of the socio-political-cultural context and cost effectiveness. Policy makers are involved throughout, and will be presented with costed policy options along with their potential consequences. Implemented options will then be evaluated. The framework represents a "policy effectiveness-feasibility loop", analogous to Tugwell's clinical effectiveness loop. The impact of this approach, and its potential generalisability, will be rigorously evaluated.

\section{SYSTEMATIC REVIEW: THE USE OF RESEARCH EVIDENCE BY PUBLIC HEALTH POLICY-MAKERS}

doi:10.1136/jech.2010.120956.52

L Orton, F Lloyd-Williams, D Taylor-Robinson, M O'Flaherty, S Capewell. Division of Public Health, School of Population Community and Behavioural Sciences, University of Liverpool, Liverpool, UK

Objective To review: the process of public health policy-making; variations in the extent of research evidence used; other influencing factors; and barriers to and facilitators of the use of research evidence.

Design Systematic review of empirical studies reporting data on policy-making in public health.

Data Sources Databases searched: MEDLINE, SCOPUS, PsychInfo, CINAHL, The Social Science Citation Index, The Science Citation Index, The Arts and Humanities Citation Index, Applied Social Sciences Index and Abstracts, Database of Reviews of Effects, Cochrane Database of Systematic Reviews, DoPHER, the Campbell Library, and the Cochrane Register of Controlled trials. Other sources: screening of organisational websites, contacting key informants and scrutinising the bibliographies of included studies.

Review methods Two reviewers independently assessed studies for inclusion; extracted data and assessed methodological quality using predesigned forms. Disagreements were resolved by consensus or by recourse to a third reviewer. Data were synthesised as a narrative review.

Results 1216 articles were retrieved. Following screening 18 studies were included: 13 qualitative studies, four surveys and one literature review. Participants included 1200 policy-makers, 72 researchers, and 174 people involved in both activities. Studies were set in a range of country and policy-making settings. Methodological quality was mixed. The process of policy-making varies widely between settings, and is viewed differently by key players. An extensive range of types of research evidence are used in policymaking. However, it has only an indirect impact and competes with many other influences. Barriers to the use of research evidence are well-described and include: policy-makers' perceptions of research evidence; the gulf between researchers and policy-makers; the culture in which policy-makers work; competing influences on policy-making; and practical constraints. Ways of overcoming these barriers are less well known, and include: research targeted at the needs of policy-makers; research clearly highlighting key messages; and capacity building. There is almost no evidence on the role of research evidence in addressing health inequalities, a key aim of public health policy.

Conclusions Action is required by both policy-makers and researchers to address the barriers identified in this systematic review. There is an urgent need for evidence on the best approaches to incorporating research evidence in public health policy, particularly that considering the complex effects on health inequalities.
053 PROTECTION AND PUBLIC HEALTH: POPULATION EVIDENCE FROM THE EU, 1980-2003

doi:10.1136/jech.2010.120956.53

${ }^{1,2} \mathrm{D}$ Stuckler, ${ }^{3} \mathrm{~S}$ Basu, ${ }^{2} \mathrm{M}$ McKee. ${ }^{1}$ Department of Sociology, Oxford University, Oxford, UK; ${ }^{2}$ London School of Hygiene and Tropical Medicine, Department of Public Health and Policy, London, UK; ${ }^{3}$ Department of Internal Medicine, University of California at San Francisco, San Francisco, California, USA

Objectives To assess the effect of social spending on population health.

Design Multivariate regression analysis was performed to investigate the relationship between age-standardised cause-specific mortality rates and social spending. Mortality data were collected from the European Health for All Database 2009 edition. Social spending per capita in purchasing-power-parity were taken from the OECD Social Expenditure Database (including family support, oldage pensions, healthcare, unemployment benefits, active labour market programmes, and support for people with disabilities). These relationships were compared with gross domestic product per capita (GDP), general government spending per capita (including prisons, education and defence), and healthcare spending per capita. Models also included controls for country- and period-fixed effects and expenditure was adjusted for inflation and purchasing power parity. Setting 13 EU countries, 1980-2003: Belgium (only to 1998), Denmark, Finland, France, Germany, Greece, Ireland, Italy, Netherlands, Spain, Sweden and the UK.

Participants $\mathrm{n} / \mathrm{a}$.

Main outcome measure Age-standardised all-cause and cause-specific mortality rates.

Results Each additional US\$100 increase in social spending per capita is significantly associated with a $0.99 \%$ reduction in all-cause, age-standardised mortality rates $(p<0.001)$. Healthcare spending per capita had no effect on all-cause mortality rates. Consistent with previous findings, we observed each US\$100 increase in GDP was associated with a $0.28 \%$ fall in all-cause mortality rates $(p<0.001)$, about one-fourth of the magnitude of the association of social welfare with mortality. After adjusting for social welfare spending, the association of GDP with all-cause mortality was reduced by over half ( $\beta=-0.11 \%, p=0.004)$. Healthcare spending was not associated with mortality. However, higher social welfare spending was significantly associated with reductions in alcohol-related deaths, cardiovascular disease and tuberculosis; findings which are biologically plausible.

Conclusion Reducing social spending could increase mortality rates. The majority of the benefits of wealth to health appears to be determined by the extent to which resources are invested in social welfare and healthcare systems. Investments in social protection seem to have greater protective effects on health than investments in health care. Stronger links between health and labour ministries offer an opportunity to take advantage of such positive synergies, especially important to protecting health during times of austerity.

\section{Ethnicity}

\section{ANOTHER HISPANIC PARADOX? THE HEALTH BENEFITS OF HISPANIC COMMUNITIES FOR NON-HISPANIC MOTHERS AND INFANT}

doi:10.1136/jech.2010.120956.54

R J Shaw, K E Pickett. Department of Health Sciences, University of York, York, UK

Background and Objectives In the US, Hispanic mothers have rates of infant mortality and low birthweight that are comparable to nonHispanic White mothers despite being more likely to live in socioeconomically deprived areas. This well known phenomenon is 